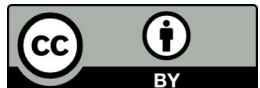

\title{
Violencia conyugal y salud familiar de la mujer en el Hospital Regional de Tumbes 2017.
}

Domestic violence and family health of the woman in the Hospital Regional de Tumbes 2017.

Violência conjugal e saúde da família da mulher no Hospital Regional de Tumbes 2017.

\section{ARTÍCULO GENERAL}

\section{Grevillí Marlitt García Godos Castillo grevilli6669@hotmail.com https://orcid.org/0000-0002-2689-7704 \\ Universidad Nacional de Tumbes, Tumbes - Perú}

\author{
Amarilis Calle Caceres \\ amariliscalle@hotmail.com \\ https://orcid.org/0000-0002-6702-8572 \\ Universidad Nacional de Tumbes, \\ Tumbes - Perú
}

Recibido 07 de Junio 2021 | Arbitrado y aceptado 25 de Agosto 2021 | Publicado en 04 Diciembre 2021

\section{RESUMEN}

A La presente investigación, le corresponde un diseño no experimental, retrospectivo, de tipo descriptivo correlacional, tuvo como objetivo general determinar la relación entre el grado de violencia conyugal y la salud de la mujer atendida en el Hospital Regional de Tumbes 2017. Para llegar a los resultados se emplearon encuestas con relación a los objetivos planteados, La población estuvo constituida por 6293 mujeres y la muestra por 90 mujeres, cada unidad muestral fue asignada al azar. Los datos obtenidos fueron analizados a través d estadística descriptiva e inferencial, Los resultados establecieron que el $39 \%$ presenta violencia conyugal en grado moderado, $33 \%$ en grado leve y $28 \%$ en grado severo. El $34 \%$ de las mujeres en estudio presentan elevada repercusión en la salud familiar, $28 \%$ severa repercusión en la salud familiar y $26 \%$ moderada repercusión $\mathrm{La}$ correlación de las variables ha demostrado que existe relación significativa entre las variables violencia conyugal y repercusión en la salud familiar grado de violencia severa ( $48 \%$ y $32 \%$ ) se asocia con repercusión elevada y severa en la salud familiar. Lo que nos permite aceptar la hipótesis planteada a través de la prueba ji cuadrado. Se ha diseñado una propuesta de intervención orientada a empoderar a las familias.

Palabras clave: mujer, familia, abuso, violencia.

\section{ABSTRACT}

This research has a non-experimental, retrospective, descriptive-correlational design, whose general objective was to determine the relationship between the degree of conjugal violence and the health of women treated at Hospital Regional de Tumbes 2017. To arrive at The results were used surveys in relation to the objectives set. The population consisted of 6293 women and the sample of 90 women, each sample unit was randomly assigned. The data obtained were analyzed through descriptive and inferential statistics. The results established that $39 \%$ present marital violence in a moderate degree, $33 \%$ in a mild degree and $28 \%$ in a severe degree. $34 \%$ of the women in the study have a high impact on family health, $28 \%$ severe impact on family health and $26 \%$ moderate impact The correlation of the variables has shown that there is a significant relationship between the variables marital violence and impact on health Family degree of severe violence $(48 \%$ and $32 \%)$ is associated with a high and severe impact on family health. This allows us to accept the hypothesis raised through the chi-square test. An intervention proposal aimed at empowering families has been designed.

Keywords: woman, family, abuse, violence.

\section{RESUMO}

A presente pesquisa tem um desenho não experimental, retrospectivo, descritivocorrelacional, cujo objetivo geral foi verificar a relação entre o grau de violência conjugal e a saúde das mulheres atendidas no Hospital Regional de Tumbes 2017. Para chegar a Os resultados foram utilizados inquéritos em relação aos objectivos traçados A população foi constituída por 6293 mulheres e a amostra por 90 mulheres, cada unidade amostral foi distribuída aleatoriamente. Os dados obtidos foram analisados por meio de estatística descritiva e inferencial.Os resultados estabeleceram que $39 \%$ apresentam violência conjugal em grau moderado, $33 \%$ em grau leve e $28 \%$ em grau grave. $34 \%$ das mulheres do estudo têm alto impacto na saúde da família, $28 \%$ impacto grave na saúde da família e $26 \%$ impacto moderado A correlação das variáveis mostrou que existe uma relação significativa entre as variáveis violência conjugal e impacto na saúde $O$ grau de violência grave na família ( $48 \%$ e $32 \%$ ) está associado a um impacto alto e grave na saúde da família. Isso nos permite aceitar a hipótese levantada por meio do teste do quiquadrado. Foi elaborada uma proposta de intervenção voltada ao empoderamento das famílias.

Palavras-chave: mulher, família, abuso, violência. 


\section{INTRODUCCIÓN}

Hablar de violencia hacia la mujer por parte de la pareja, es remontarnos a un fenómeno tan antiguo como la humanidad misma, pero que sin embargo se está visualizando actualmente con tal magnitud, que la posicionan como problema social y de salud pública importante. La violencia contra la mujer en relación conyugal, en el interior de las familias, es capaz de producir efectos adversos en la victima y en los miembros de la familia. Una de cada tres mujeres sufre violencia en alguna etapa de su vida, considerándola una "pandemia silenciosa" y "epidemia invisible", afirman que se incluirá como una de las primeras quince causas de enfermedad y muerte para el año $2020^{1,2,3}$. "La organización mundial de la salud (OMS 2003)4, argumenta que es responsable del 5 a 16\% de los años de vida saludables perdidos; un año perdido por cada cinco años de vida saludable. Es la primera causa de daño físico en el grupo de 15 a 44 años de edad y se le atribuye ser la causa de un descenso del $12,3 \%$ del producto bruto interno (PBI) mundial”.

Las cifras de la violencia en el mundo, han aumentado a pasos agigantados en relación a la década de los noventa, según "El Banco mundial, la OMS y la Comisión de los derechos humanos; ya que se manifiestan cifras que van desde 10 hasta 69\%" 5, 6. El Perú, en el año 2016 presento una alta prevalencia de violencia contra la mujer, llegando al feminicidio (124 casos y 258 tentativas). Los casos reportados de violencia fueron 621 187, de los cuales el 50,5\% fue violencia psicológica, 39,6\% violencia física y 9,5\% violencia sexual. Tumbes no es ajeno a esta problemática habiéndose reportado en el mismo año 2 feminicidios y 1 tentativa, además 947 casos de violencia, 55\% de tipo psicológico, $44 \%$ de tipo física y $1 \%$ sexual $^{7}$.

La victimización contra la mujer, en relación conyugal, es un fenómeno que se agrava a gran escala y ocupa titulares en los medios de comunicación, sin distinguir entre clases sociales, cultura, edad, religión o país. Ser una más implica vivir constantemente bajo la amenaza existente o anticipada de ser violentada, estableciendo en la mayoría de casos, un escenario de estrés crónico, por la persistencia de la misma ${ }^{8,4}$. Esta situación produce efectos devastadores para la salud, no solo de la mujer víctima de maltrato, sino también para los integrantes de la familia, con resultados a lo largo de la vida. Independientemente del escenario donde se ejerza, tiene efectos graves a nivel personal, en el entorno familiar y social. Por tanto la familia que vive bajo estrés constante, es un grupo vulnerable para sufrir desequilibrios en su ciclo vital y en su funcionalidad ${ }^{9}$. 
Esta situación que ocupa los titulares de muchos medios de comunicación, se observa en los diferentes escenarios, siendo uno de ellos el Hospital Regional de Tumbes, donde diariamente acuden en busca de asistencia, muchas mujeres víctimas de violencia, producidas en diferentes circunstancias, pero básicamente en el ámbito familiar, mujeres, la mayoría de ellas, con una actitud de sumisión e incluso de aceptación de la violencia como natural, situación al parecer generada en un escenario e interacciones e interrelaciones al interior de las familias, y que no siempre son tomadas en cuenta para un abordaje integral del problema.

Por todo lo expuesto, el problema de investigación se ha planteado por la necesidad de establecer ¿Cuál es la relación entre violencia conyugal y salud familiar de la mujer atendida en el Hospital Regional de Tumbes 2017?, es un tema de actualidad que se ha constituido en motivo de investigación, por la implicancia que tiene en la salud de la mujer y su familia y que va en aumento afectando a diferentes grupos poblacionales, de los cuales la mujer es uno de los más vulnerables. El estudio es altamente relevante y ha encontrado justificación en la medida que las graves consecuencias que derivan de la violencia han sido reconocidas por la OMS y OPS, calificándola como un atentado contra el desarrollo humano, la integridad y los derechos humanos, situación que la constituye en un grave problema de salud pública, cultural y generacional por lo que encabeza su agenda prioritaria.

La problemática de la violencia, además tiene impacto social, sus alcances involucran el plano teórico y práctico, y es que a partir de los resultados se tiene una aproximación objetiva y mayor comprensión del grado de violencia contra la mujer y el grado de repercusión en la salud familiar en nuestra propia realidad, constituyendo éste, el valor teórico de la investigación. Mientras que en el plano práctico, permitirá ser un referente para orientar y mejorar la respuesta sanitaria, política y social, no sólo para la identificación de los casos de violencia sino también para la intervención integral, que mejore la calidad de vida de estas mujeres, se erradique la violencia y los patrones socioculturales que la perpetúan; mediante planes de trabajo con perspectivas transformadoras y preventivas, con enfoque intersectorial y transdisciplinarios, que involucre a la sociedad civil y la participación comunitaria.

A partir de lo señalado, los directamente beneficiados no sólo serán las mujeres y sus familias, sino también la comunidad. La hipótesis que sustenta el trabajo es: Existe relación entre la violencia conyugal y la salud familiar de la mujer en el Hospital Regional de Tumbes 2017, siendo el objetivo general: determinar la relación entre la violencia 
conyugal y salud familiar de la mujer en el Hospital Regional de Tumbes 2017. Los objetivos específicos: identificar la violencia conyugal en grados: severa, moderada y leve, identificar el nivel de repercusión de la violencia y establecer la relación entre variables.

\section{METODOLOGIA}

La investigación se desarrolló a través de un diseño no experimental, con enfoque metodológico cuantitativo, retrospectivo, de tipo correlacional y de corte transversal. El universo estuvo constituido por 6293 mujeres en edad fértil, la muestra se calculó en 90, se seleccionaron a través de muestreo aleatorio simple por balotas, sorteando 6 usuarias diariamente, hasta completar la muestra.

La recolección de datos se hizo con una encuesta, los Instrumentos fueron validados en realidades similares, a través de una prueba piloto, se aplicó el Coeficiente de Alfa de Cronbach com resultado 0,9 (90\%) de confiabilidad y validez. Se solicitó el consentimiento informado, luego se aplicaron los instrumentos: cuestionario de tamizaje para violencia contra la mujer que permitió definir los casos de violencia según tipo y grado, extraído del Proyecto rompiendo silencio del instituto de la mujer Lana Cáceres de Arequipa Perú y el de repercusión familiar (IRFA), Consta de 20 ítems, que evalúan el estado de salud de los miembros de una familia ante la convivencia con un evento estresante. Los datos se transfirieron a una base de datos, en el programa Excel y SPSS versión 21 organizándose en tablas y figuras estadísticas. La interpretación, análisis y discusión de resultados, se realizó a través de estadística descriptiva e inferencial y la prueba no paramétrica Ji cuadrado. Respecto al código de ética sustentada en el informe Belmont, estos fueron: beneficencia, confidencialidad y justicia.

\section{RESULTADOS Y DISCUSIÓN}

Tabla 01. Grado de violencia conyugal de la mujer en el Hospital José Alfredo Mendoza Olavarria Tumbes, 2017.

TIPO DE VIOLENCIA VIOL. PSICOLOGICA VIOLENCIA SEXUAL VIOLENCIA FISICA

GRADO DE VIOLENCIA

o
$\mathrm{N}$

$\%$

○

$\mathrm{N}$

$\%$

$\circ$ 


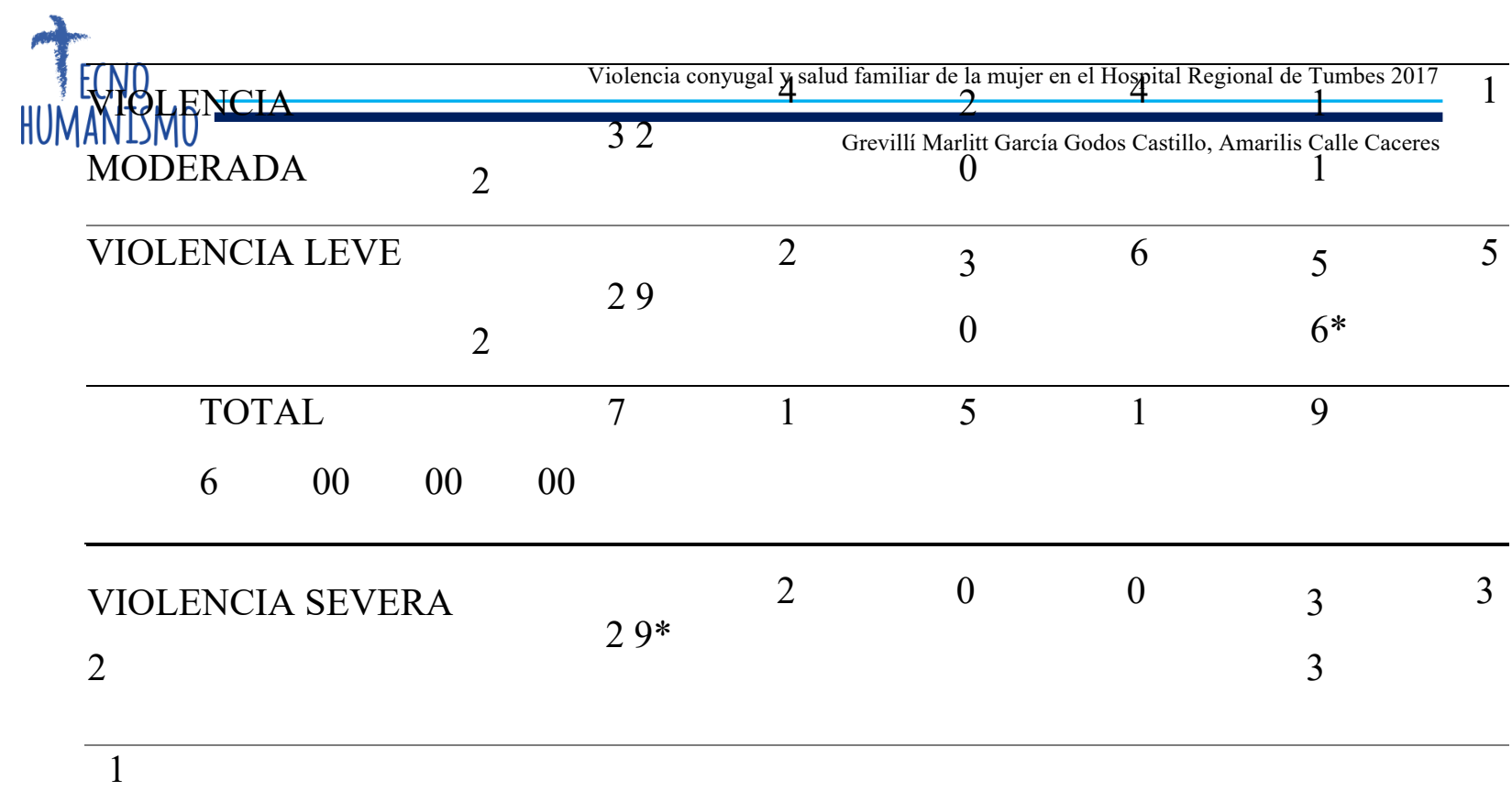

Fuente: Encuesta para detección del grado de violencia. Utilizada en el estudio violencia conyugal y salud familiar de la mujer en el Hospital Regional Tumbes 2017.

${ }^{*} \mathrm{p}<0.005$

Del total de las mujeres en estudio, el $39 \%$ presenta violencia conyugal en grado moderado, $33 \%$ en grado leve y $28 \%$ en grado severo.

Tabla $02 \quad$ Nivel de repercusión en la salud familiar de la mujer en el Hospital José Alfredo Mendoza Olavarria Tumbes, 2017.

TIPO DE VIOLENCIA VIOL. PSICOLOGICA
VIOLENCIA FISICA
NIVEL DE N $\%$ N $\%$ N $\%$ REPERCUSION $\circ \circ \circ$

NO REPERC

LEVE REPERC

MODERADAREPERC

ELEVADAREPERC
3

5

12

7

$24^{*}$

4

0

0

0

0

7

0

0

0

0

2

1
0

2

5

6*

3

6

2

0

2 


\begin{tabular}{clllllllll}
\hline SEVERAREPERC & & & & 3 & 1 & 2 & 2 & 2 \\
& & & & $23 *$ & & 0 & & 2 \\
\hline TOTAL & 1 & 5 & 1 & 9 & 1 & & & \\
6 & 700 & 00 & 00 & & & & & & \\
\hline
\end{tabular}

Fuente: Encuesta para detección del grado de violencia. Utilizada en el estudio violencia conyugal y salud familiar de la mujer en el Hospital Regional de Tumbes 2017. $\mathrm{p}>0.05$

El $34 \%$ de las mujeres en estudio presentan elevada repercusión en la salud familiar, $31 \%$ severa repercusión en la salud familiar y 26 \% moderada repercusión.

Tabla $03 \quad$ Nivel de repercusión en la salud familiar y grado de violencia conyugal e la mujer en el Hospital José Alfredo Mendoza Olavarria Tumbes, 2017.

$\begin{array}{lcl}\text { GRADO } & \text { DE VIOLENCIA SEVERA } & \text { VIOLENCIA } \\ \text { VIOLENCIA } & \text { MODERADA } & \text { LEVE }\end{array}$

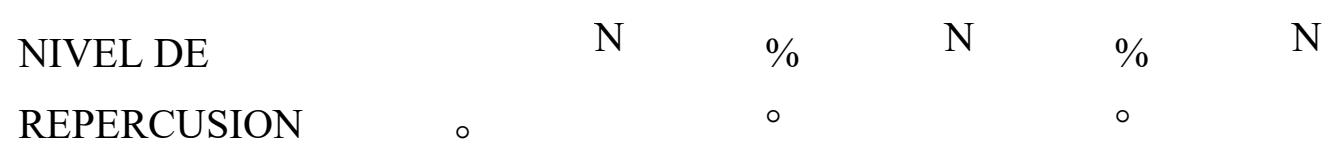

NO REPERCUSION

1

4

1

3

2

6

2

REPERCUSION

MODERADA

96

$18^{*} \quad 43 \quad 17^{*} \quad 60$


REPERCUSION SEVERA

$82 * \quad 30$
19

20

\section{TOTAL}

5

$\%$

Fuente: Encuesta para detección del grado de violencia. Utilizada en el estudio violencia conyugal y Salud familiar de la mujer en el Hospital Regional de Tumbes 2017. $* \mathrm{p}<0.05$

Al establecer la relación entre las variables se observa que el grado de violencia severa se asocia con repercusión elevada y severa (48\% y $32 \%)$ en la salud familiar.

La violencia contra la mujer, es un problema mundial con graves repercusiones en la salud de las personas que la viven, se considera que los estudios sobre ella hasta ahora expuestos son apenas el inicio del conocimiento sobre un fenómeno que afecta el desarrollo de los pueblos. En la tabla 01, el 39\% de mujeres presenta violencia conyugal en grado moderado, $33 \%$ en grado leve y $28 \%$ en grado severo.

Los actos violentos se naturalizan siguiendo las lógicas de un ejercicio del poder de los de arriba contra los que se encuentran en posiciones de debilidad, en ese sentido los agresores responsabilizan a las victimas alegando infidelidad, celos y negativa a continuar con la relación. Al respecto, Ortiz, Cañavera y Alcántara, coinciden que el grado severo de la violencia está presente en familias con desequilibrios de funcionalidad, con características de dependencia hacia las drogas, tienen alta inadaptabilidad, siendo la exposición al maltrato de la madre cualquiera sea la intensidad de ésta y el tipo, afectando a los miembros de la familia en aspectos psicológicos y somáticos ${ }^{10,11,12}$. García Godos sugiere que el grado de violencia contra la mujer en Tumbes es severo, siendo las relaciones estresantes en el interior de las familias ${ }^{13}$.

La violencia se manifiesta en más de una forma, pudiéndose perpetrar más de un tipo a la vez, además el daño a la salud no solo depende del tipo o forma de la violencia infringida sino también por la intensidad y el grado en que la recibe, tanto la víctima como los que viven a su alrededor. Esta situación se agrava cuando no se recibe ayuda externa, como apoyo emocional, material, afectivo y social. 
En la tabla 02, El $34 \%$ de las mujeres presentan elevada repercusión en la salud familiar, $31 \%$ severa repercusión en la salud familiar y $26 \%$ moderada repercusión, al respecto se puede afirmar que la salud familiar en nuestra realidad no es óptima, como lo sugiere la OMS, pudiendo deberse a algún grado de violencia con la que se convive en el interior de las familias, hecho que para muchos sigue siendo natural ya que socialmente a los hombres se les prepara para asumir un rol dominante, vinculado al poder y a la autoridad ${ }^{4}$. Por el contrario, en las mujeres se han valorado rasgos como la dulzura, la pasividad y la obediencia, así como la capacidad para expresar emociones.

Es evidente que la violencia en relación de pareja, en el interior de la familia repercute de forma negativa en la salud, independientemente del tipo y magnitud de violencia que se ejerza. Sobre el particular Brito et. al , Carriera y González, señalan que más del 80 \% de mujeres sufrió almenos un episodio violento en su vida de pareja y coinciden al aportar que las familias no funcionales tienen una mayor incidencia de enfermos crónicos y plantean que la adicción y problemas de conducta de sus miembros son frecuentes ${ }^{14,15}$, 16. Hallazgos que reafirman a la violencia como un problema importante de salud pública.

En la tabla 03, Al establecer la relación entre las variables se observa que el grado de violencia severa (48\% y $32 \%$ ) se asocia con repercusión elevada y severa en la salud familiar. Sobre el particular, Tarragona y Alvarado, hacen alusión a la repercusión de la violencia en la salud y coinciden con el estudio al afirmar que la repercusión esta en relación al grado de violencia perpetrada ${ }^{17,18}$. Esta asociación nos permite plantear que a mayor grado de violencia mayor nivel de repercusión en la salud familiar. Aunque hay posiciones teóricas que indican que la violencia independientemente del grado ya general implicancias en la salud de las víctimas.

\section{CONCLUSIONES}

Del total de las mujeres en estudio, el 39\% presenta violencia conyugal en grado moderado, $33 \%$ en grado leve y $28 \%$ en grado severo. La salud familiar se afectó de la siguiente forma: el $34 \%$ de las mujeres presentan elevada repercusión en la salud familiar, $31 \%$ severa repercusión en la salud familiar y $26 \%$ moderada repercusión. Al establecer la relación entre las variables se observa que el grado de violencia severa ( $48 \%$ y $32 \%$ ) se asocia con repercusión elevada y severa en la salud familiar.

\section{REFERENCIAS BIBLIOGRÁFICAS}


Ferrer P. VA, Bosch Fiol E, Ramis Palmer MC. La formación de los/as profesionales de la salud para afrontar la violencia contra las mujeres en la pareja. Clínica y Salud.

Seriado en internet]. 2008[citado 18 junio 2009]; 19 (1): 59-81. Disponible en:

http://redalyc.uaemex.mx/redalyc/pdf/1806/180613876003.pdf

UNIFEM. Violence against Women. [Página Principal de un sitio Web]. New York:

UNIFEM; 2008 [citado12 junio 2009]. Disponible en: http://www.unifem.org/gender_issues/violence_against_women/

Moreno J, Delgado E, Guía de recursos para mujeres víctima de violencia. Granada:

Consejería de la mujer; 2003.

Organización Mundial de la Salud (OMS), en el informe mundial sobre la violencia y la salud (2003), OPS/OMS. Informe Mundial sobre Violencia y Salud. Washington, 2002.

Pérez P. R. Protocolo Agencial de Intervención Integrada con Víctimas / Sobrevivientes de Violencia Domestica. [Monografía en Internet]. San Juan: Departamento de Salud; 2002 [citado 17 diciembre 2009]. Disponible en: http://www.salud.gov.pr/Services/ProtocolosMedicos/Protocolos/Protocolo\%20agen cial\%20del\%20DS \%20con\%20VD.pdf

Organización Panamericana de la Salud. La violencia basada en el género: un problema de salud pública y de derechos humanos. Revista Futuros. [Seriado en Internet] 2005 [citado 2 septiembre 2009]; 3 (10): 1-4. Disponible en: http://www.revistafuturos.info//raw_text_Fraw_futuro/Fviol_mujer.doc.

Centro de Emergencia Mujer (CEM) 2017. Violencia contra la mujer en el Perú.

López A. L. Guía metodológica para la asistencia a mujeres víctimas de violencia. Rev Electr MEDISUR. [Seriado en Internet]. 2009 [citado 11 noviembre 2009]; 7(5):

[aprox. 18 p.]. Disponible en:

http://www.medisur.sld.cu/index.php/medisur/article/viewArticle/805/5483

Ruíz I, Blanco P, Vives C. Violencia contra la mujer en la pareja: determinantes y respuestas sanitarias. Gac Sanit. [Seriado en internet]. 2004 [citado19 junio 2009[;18(supp12):[aprox.18p.]. Disponible en:

http://scielo.isciii.es/scielo.php?pid=S0213- $\quad 112004000500003 \&$ script http://www.medisur.sld.cu/index.php/medisur/article/viewArticle/805/5483

Ortiz MT. Violencia intrafamiliar desde la perspectiva de la mujer [Tesis para la obtención al grado de Especialista en Psicología de la Salud]. Ciudad de La Habana, 2000. Cañavera M. Díada Marital Disfuncional y Fármaco Dependencia. [Tesis Bachiller]. Universidad la católica Lima; 1988. 
Alcántara L., Las victimas invisibles afectación psicológica de menores expuestos a Violencia de género. [Tesis doctoral online] Murcia: España Universidad de Murcia; 2010. [Accesado diciembre 2018]. Disponible en URL: file://D:/E/tesis\%20doctoral/grado\%20de\%20violencia.pdf

García - Godos C G. caracterización del sistema familiar y violencia contra la mujer. Tumbes distrito 2011.[Tesis para la obtención al grado de Magister en salud comunitaria].Escuela de post grado. 2012.

Brito-Tinajero C. y Delgadillo L., Relación entre violencia de pareja y estrés parental en población Mexicana. [Revista online] Centro de Investigación de la Universidad La Salle: México; 2015. Vol. 12, No. 45, enero-junio, 2016: 91-106 [accesado noviembre 2018] disponible en URL: http://ojs.dpi.ulsa.mx/index.php/rci/

Carriera D E. Comportamiento biosocial de las familias de la comunidad de un consultorio médico. Policlínico Docente Playa [Tesis para la obtención al grado de especialista en Medicina General Integral]. Ciudad de La Habana, Facultad Finlay Albarrán, 1990.

González G J. Factores de riesgo familiar en el intento suicida. Municipio Playa. 1995

[Tesis para la obtención al grado de Master en Psicología de la Salud].Facultad de Salud Pública, 1996.

Tarragona Oriols M, Historia de victimización materna y su impacto sobre la salud mental de los menores en entornos de violencia de pareja. [Tesis doctoral online] Bellaterrra: Barcelona España. Universidad autónoma de Barcelona; 2016. [Accesado 12 de 2018]. Disponible en URL: file:///D:/E/tesis\%20doctoral/conclusiones.pdf Alvarado de Rattia E. Percepción de exposición a violencia familiar en adolescentes de población general: consecuencias para la salud, bajo un enfoque de resiliencia. [Tesis doctoral online] Madrid: España. Universidad. Universidad Complutense de Madrid Facultad de psicología; 2013 [Accesado diciembre 2018]. Disponible en URL: http://eprints.ucm.es/21595/1/T34520.pdf 